\title{
Mitral annulus is enlarged and functionally impaired in adult patients with repaired tetralogy of Fallot as assessed by three-dimensional speckle-tracking echocardiography-results from the CSONGRAD Registry and MAGYAR-Path Study
}

\author{
Attila Nemes ${ }^{1}$, Árpád Kormányos ${ }^{1}$, Kálmán Havasi ${ }^{1}$, Zsolt Kovács ${ }^{2}$, Péter Domsik ${ }^{1}$, Anita Kalapos ${ }^{1}$, \\ István Hartyánszky ${ }^{1}$, Nóra Ambrus ${ }^{1}$, Tamás Forster ${ }^{1}$ \\ ${ }^{1}$ 2nd Department of Medicine and Cardiology Centre, Medical Faculty, Albert Szent-Györgyi Clinical Center, University of Szeged, Szeged, \\ Hungary; ${ }^{2}$ Department of Cardiology, Szent Rókus Hospital, Baja, Hungary \\ Contributions: (I) conception and design: A Nemes, Á Kormányos, K Havasi, T Forster; (II) administrative support: N Ambrus; (III) provision of study \\ materials or patients: K Havasi, I Hartyánszky; (IV) collection and assembly of data: Á Kormányos, A Kalapos, P Domsik, Z Kovács; (V) data analysis \\ and interpretation: Á Kormányos, A Nemes; (VI) manuscript writing: all authors; (VII) final approval of manuscript: all authors. \\ Correspondence to: Attila Nemes, MD, PhD, DSc, FESC. 2nd Department of Medicine and Cardiology Center, Medical Faculty, Albert Szent-Györgyi \\ Clinical Center, University of Szeged, H-6725 Szeged, Semmelweis Street 8, Hungary. Email: nemes.attila@med.u-szeged.hu.
}

\begin{abstract}
Background: Fibrous mitral annulus (MA) is an important part of the mitral valve having a role in forwarding blood from the left atrium (LA) to the left ventricle (LV). MA can be assessed by threedimensional speckle-tracking echocardiography (3DSTE) respecting the cardiac cycle. The present study was designed to test whether repaired Tetralogy of Fallot (TOF) is associated with morphological and functional alterations of the MA. The role of the type of treatment (early total reconstruction vs. early palliation, late correction) was also assessed.

Methods: The study population consisted of 29 consecutive adults repaired TOF patients (mean age: $35.4 \pm 15.5$ years, 18 men), from which 13 patients underwent early total reconstruction (etrTOF), while 16 patients were firstly palliated and later corrected (pcTOF). Their data were compared to that of 76 age- and gender-matched healthy controls (mean age: $35.9 \pm 7.6$ years, 33 men). All repaired TOF patients and controls were assessed by two-dimensional (2D) Doppler echocardiography and 3DSTE.

Results: Dilated end-systolic and end-diastolic MA diameter, area and perimeter and reduced MA fractional area change and MA fractional shortening could be demonstrated in repaired TOF patients as compared to controls. Increased body surface area-indexed end-diastolic and end-systolic MA diameter and perimeter could be demonstrated in pcTOF patients as compared to that of etrTOF cases.

Conclusions: MA enlargement and functional impairment could be detected in adult patients with repaired TOF regardless of the type of correction. However, pcTOF patients have worse results.
\end{abstract}

Keywords: Mitral annulus (MA); function; three-dimensional (3D); echocardiography; speckle-tracking

Submitted Mar 27, 2019. Accepted for publication Jun 12, 2019.

doi: 10.21037/cdt.2019.06.08

View this article at: http://dx.doi.org/10.21037/cdt.2019.06.08

\section{Introduction}

Tetralogy of Fallot (TOF) is a cyanotic congenital heart disease (CHD) consisting of ventricular septal defect, overriding aorta, pulmonary stenosis, and right ventricular hypertrophy $(1,2)$. Due to the opportunity of early total reconstruction, there is an increasing number of patients with repaired TOF in the adult clinical practice (1). Several echocardiographic methods can be used for 
their assessment including three-dimensional speckletracking echocardiography (3DSTE), which is a novel echocardiographic modality with capability of $3 \mathrm{D}$ volumetric and functional assessment of heart chambers (3-7).

Fibrous mitral annulus (MA) is an important part of the mitral valve having a role in forwarding blood from the left atrium (LA) to the left ventricle (LV) and can be assessed respecting the cardiac cycle by 3DSTE (8). The present study was designed to test whether repaired TOF is associated with morphological and functional alterations of the MA as compared to that of age- and gender matched healthy controls. The role of the type of treatment (early total reconstruction $v s$. early palliation, late correction) was also assessed.

\section{Methods}

\section{Patient population}

More than 3,000 patients with CHD have been involved into the Registry of $\mathrm{C}(\mathrm{S}) \mathrm{ONGenital}$ caRdiAc Disease patients at the University of Szeged (CSONGRAD Registry), who have been treated and/or operated on since 1961 at the Department of Pediatrics, Department of Cardiac Surgery, and 2nd Department of Medicine and Cardiology Center were collected "Csongrád" is the name of one of the 19 Hungarian counties where the city of Szeged is placed) (9). From this patient population, 29 consecutive adults repaired TOF patients (mean age: $35.4 \pm 15.5$ years, 18 men) were recruited, from which 13 patients underwent early total reconstruction (etrTOF), while 16 patients were firstly palliated and later corrected (pcTOF). Early total reconstruction was performed at the age of $5.3 \pm 3.2$ years in etrTOF patients (mean follow-up period: $29.0 \pm 12.0$ years). Blalock-Taussig operation $(n=15)$, Brock surgery $(n=2)$, Brock surgery and Blalock-Taussig operation $(n=1)$ were performed as a palliation in pcTOF patients at the age of $4.4 \pm 4.2$ years. Late correction was performed in pcTOF patients at the age of $12.6 \pm 13.1$ years (mean follow-up period was $33.7 \pm 14.2$ years; from early palliation to late correction it proved to be $25.0 \pm 11$.6 years). Their data were compared to that of 76 age- and gendermatched adult healthy controls (mean age: $35.9 \pm 7.6$ years, 33 men). All repaired TOF patients and controls were in sinus rhythm and assessed by two-dimensional (2D) Doppler echocardiography and 3DSTE. A special study was organized at the Cardiology Center of the University of Szeged to assess diagnostic and prognostic significance of 3DSTE-derived parameters named MAGYAR-Path Study (Motion Analysis of the heart and Great vessels bY three-dimensionAl speckle-tRacking echocardiography in Pathological cases). All repaired TOF patients and control subjects gave informed consent. The institutional human research committee approved the study which complied with the 1975 Declaration of Helsinki.

\section{D Doppler and tissue Doppler echocardiography}

Toshiba Artida ${ }^{\mathrm{TM}}$ echocardiographic device (Toshiba Medical Systems, Tokyo, Japan) with a PST-30SBP phasedarray transducer was used for routine 2D Doppler and tissue Doppler echocardiography in all patients. LA and LV dimensions and ejection fraction were assessed according to the guidelines. Doppler echocardiography was used to exclude valvular regurgitations and stenoses as well as for the measurement of early and late diastolic mitral inflow velocities and their ratio.

\section{D speckle-tracking echocardiography}

For 3DSTE-derived data acquisitions, the same Toshiba Artida $^{\mathrm{TM}}$ echocardiography machine (Toshiba Medical Systems, Tokyo, Japan) using a PST-25SX matrix-array transducer from apical window was used (10). A fullvolume pyramid-shaped $3 \mathrm{D}$ dataset was created by the software from separately acquired six wedge-shaped subvolumes during a single breath-hold and constant RR interval on ECG. 3D analysis was performed by $3 \mathrm{D}$ Wall Motion Tracking software version 2.7 (Toshiba Medical Systems, Tokyo, Japan). For analysis of MA, a virtual 3D model of the $\mathrm{LV}$ was generated by the help of automatically selected apical two- (AP2CH) and fourchamber $(\mathrm{AP} 4 \mathrm{CH})$ views and 3 short-axis views at basal, midventricular and apical LV levels at end-diastole. Following optimization of image planes on the endpoints of the MA on $\mathrm{AP} 2 \mathrm{CH}$ and $\mathrm{AP} 4 \mathrm{CH}$ views, several measurements were performed on the $\mathrm{C} 7$ short-axis view (Figure 1) (8):

(I) For morphological assessment of MA, its diameter (MAD), area (MAA) and perimeter (MAP) were measured both at end-diastole and end-systole. MAD was defined as the perpendicular line drawn from the peak of MA curvature to the middle of the straight MA border. MAA and MAP were assessed by planimetry;

(II) For functional assessment of MA, several functional 


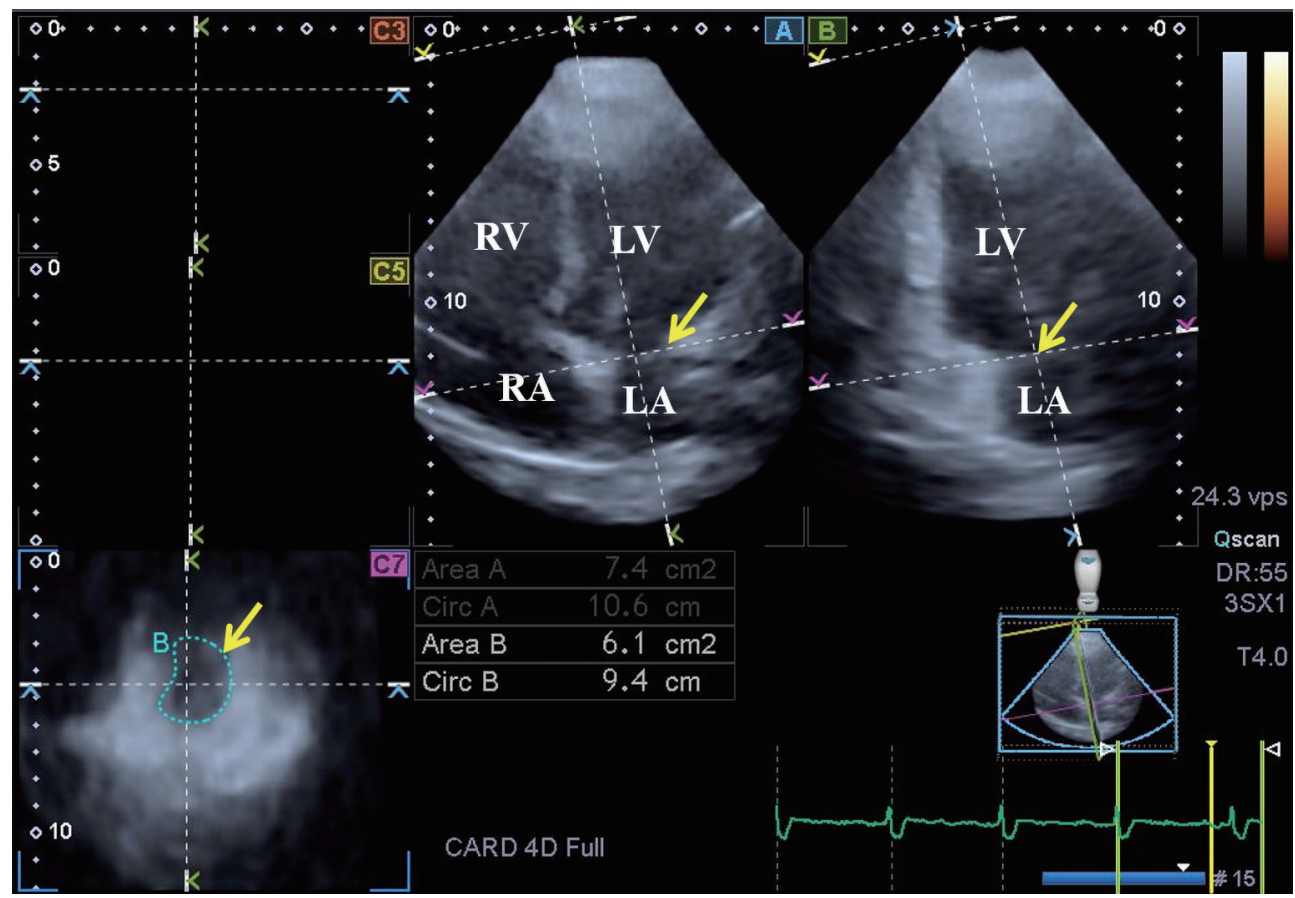

Figure 1 Images from a 3D full-volume dataset presenting the MA in a patient with repaired TOF: (A) Apical four-chamber view; (B) apical two-chamber view and a cross sectional view at the level of the MA (C7) optimized on apical four- and two-chamber views. The yellow arrow represents the mitral annular plane on the long- (A,B) and short-axis (C7) images. MA, mitral annulus; TOF, tetralogy of Fallot; LA, left atrium; LV, left ventricle; RA, right atrium; RV, right ventricle.

properties were calculated using MAD and MAA data: MA fractional shortening $($ MAFS $)=($ end diastolic MAD - end-systolic MAD)/end-diastolic MAD $\times 100$ and MA fractional area change $($ MAFAC $)=($ end-diastolic MAA - end-systolic MAA)/end-diastolic MAA $\times 100$.

\section{Statistical analysis}

Two-tailed statistical tests were used in all comparisons and a statistical significance was defined with a $\mathrm{P}$ value $<0.05$. Continuous data were presented as mean values \pm standard deviation, while categorical data were summarized as a count and percentage. For categorical variables, Fisher's exact test was used. For continuous variables, ShapiroWilk test was used to test the normal distribution and if it was confirmed, Student $t$-test was used. If not normally, normally distributed datasets were compared, MannWhitney-Wilcoxon test was used. Correlations were assessed by calculating Pearson's correlation coefficients. Data were analysed using Medcalc software (MedCalc, Mariakerke, Belgium).

\section{Results}

\section{Demographic and 2D echocardiographic data}

Enlarged left atrial diameter and normal LV dimensions and volumes with preserved $\mathrm{LV}$ ejection fraction were found in repaired TOF patients regardless of the type of correction as compared to controls (Table 1). Tricuspid annular plane systolic excursion $(18.4 \pm 4.8$ vs. $16.7 \pm 3.5 \mathrm{~mm}, \mathrm{P}=\mathrm{ns})$ and right ventricular fractional area change $(37.1 \% \pm 21.7 \% v s$. $43.4 \% \pm 10.6 \%, \mathrm{P}=\mathrm{ns})$ did not differ between etrTOF and pcTOF patients. Valvular regurgitations and the ratio of their grades are presented in Table 1.

\section{D speckle-tracking echocardiography}

Dilated end-systolic and end-diastolic MAD, MAA and MAP could be demonstrated in repaired TOF patients as compared to controls (Table 2). MAFAC and MAFS were reduced regardless of the type of treatment. Both BSA-indexed and non-indexed end-systolic MAD, MAA, and MAP were significantly increased in etrTOF patients as compared to that of healthy controls. Almost all BSA-indexed and non- 
Table 1 Baseline demographic and 2D Doppler echocardiographic data in patients with repaired TOF and controls

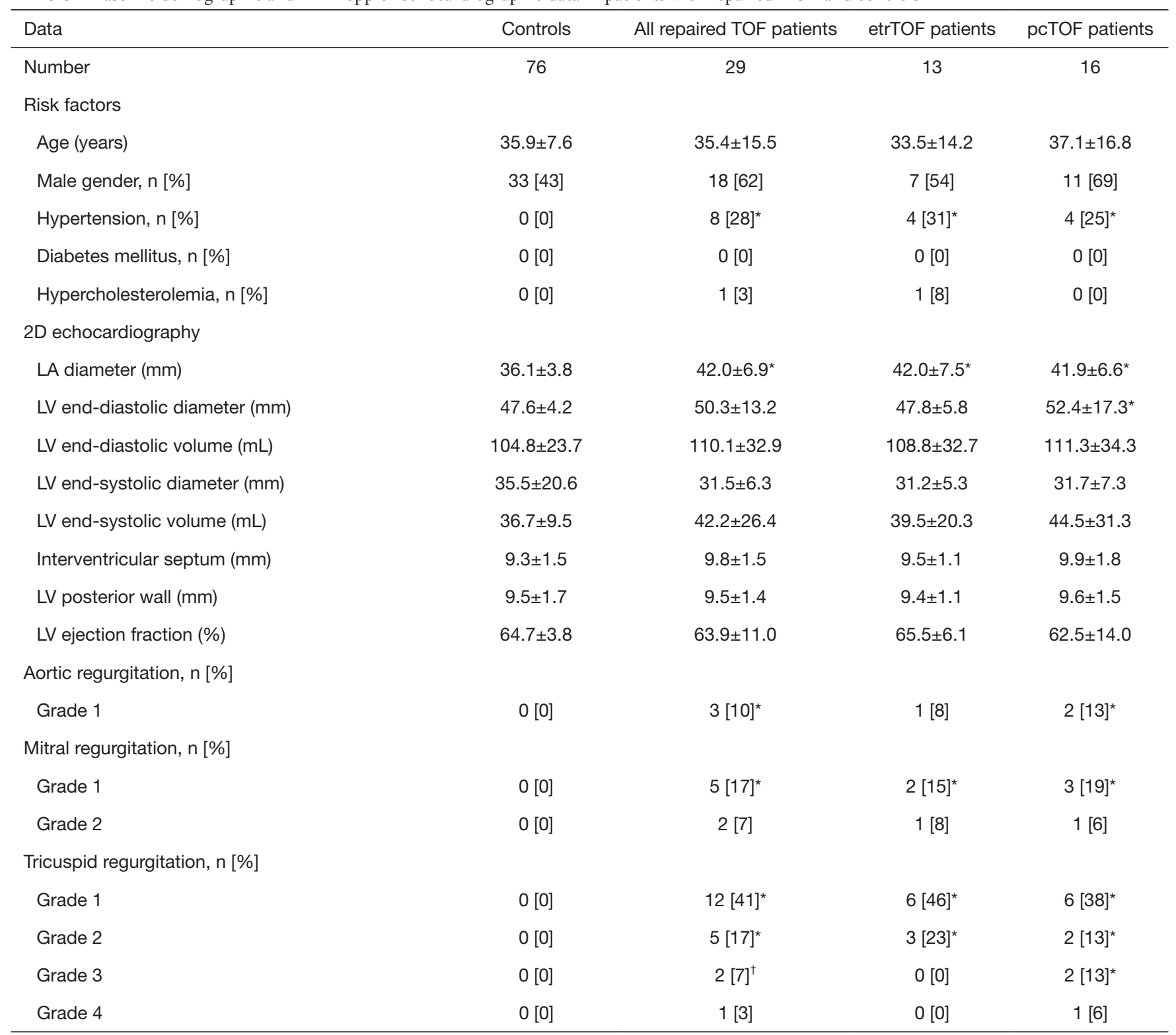

*, $\mathrm{P}<0.05$ vs. Controls; ${ }^{\dagger}, \mathrm{P}=0.07$ vs. Controls. LA, left atrial; LV, left ventricular; TOF, tetralogy of Fallot; etrTOF, TOF early total reconstruction; pcTOF, TOF early palliation, late correction.

indexed MA parameters were enlarged in pcTOF patients as compared to controls. Increased BSA-indexed end-diastolic and end-systolic MAD and MAP could be demonstrated in pcTOF patients as compared to that of etrTOF cases.

\section{Correlations}

MA functional properties (MAFAC, MAFS) did not correlate with 3DSTE-derived LV-EF neither in controls, nor in repaired TOF patients. MAA-S and MAP-S showed correlations with age at the time of the total reconstruction in etrTOF patients $(\mathrm{r}=0.57, \mathrm{P}=0.04$ and $\mathrm{r}=0.57, \mathrm{P}=0.04$, respectively). The other MA parameters did not show correlations with ages. Similar relationships between ages at the time of early palliation, late correction or difference between these ages and MA dimensions and functional 
Table 2 Comparison of 3D speckle-tracking echocardiography-derived mitral annular morphological and functional parameters between patients with repaired TOF and controls

\begin{tabular}{|c|c|c|c|c|}
\hline Data & Controls & All repaired TOF patients & etrTOF patients & pcTOF patients \\
\hline MAD-D (cm) & $2.47 \pm 0.40$ & $2.65 \pm 0.42^{*}$ & $2.62 \pm 0.44$ & $2.68 \pm 0.41$ \\
\hline $\mathrm{MAD}-\mathrm{D} / \mathrm{BSA}\left(\mathrm{cm} / \mathrm{m}^{2}\right)$ & $1.34 \pm 0.22$ & $1.48 \pm 0.23^{*}$ & $1.40 \pm 0.16$ & $1.59 \pm 0.25^{\star} \dagger$ \\
\hline MAA-D $\left(\mathrm{cm}^{2}\right)$ & $7.62 \pm 2.21$ & $8.93 \pm 3.09^{*}$ & $8.49 \pm 3.03$ & $9.29 \pm 3.18^{\star}$ \\
\hline MAP-D (cm) & $10.41 \pm 1.50$ & $11.35 \pm 1.93^{*}$ & $11.05 \pm 1.91$ & $11.60 \pm 1.98^{\star}$ \\
\hline MAP-D/BSA $\left(\mathrm{cm} / \mathrm{m}^{2}\right)$ & $5.63 \pm 0.82$ & $6.29 \pm 1.03^{*}$ & $5.88 \pm 0.74$ & $6.80 \pm 1.08^{*} \dagger$ \\
\hline MAD-S (cm) & $1.66 \pm 0.42$ & $2.16 \pm 0.38^{*}$ & $2.08 \pm 0.42^{*}$ & $2.22 \pm 0.35^{\star}$ \\
\hline MAD-S/BSA $\left(\mathrm{cm} / \mathrm{m}^{2}\right)$ & $0.90 \pm 0.23$ & $1.19 \pm 0.22^{*}$ & $1.09 \pm 0.17^{\star}$ & $1.30 \pm 0.21^{\star} \dagger$ \\
\hline MAP-S/BSA $\left(\mathrm{cm} / \mathrm{m}^{2}\right)$ & $3.83 \pm 0.64$ & $5.36 \pm 0.86^{\star}$ & $5.01 \pm 0.67^{\star}$ & $5.78 \pm 0.89^{*} \dagger$ \\
\hline \multicolumn{5}{|c|}{ Functional parameters, \% } \\
\hline MAFAC & $52.25 \pm 14.24$ & $27.60 \pm 13.50^{\star}$ & $29.38 \pm 13.75^{\star}$ & $26.14 \pm 13.56^{\star}$ \\
\hline MAFS & $31.98 \pm 15.66$ & $18.25 \pm 10.56^{*}$ & $20.45 \pm 10.40^{*}$ & $16.47 \pm 10.68^{\star}$ \\
\hline
\end{tabular}

${ }^{*}, \mathrm{P}<0.05$ vs. Controls; ${ }^{\dagger}, \mathrm{P}<0.05$ vs. etrTOF. MAA-D, end-diastolic mitral annular area; MAA-S, end-systolic mitral annular area; MAD-D, end-diastolic mitral annular diameter; MAD-S, end-systolic mitral annular diameter; MAFAC, mitral annular fractional area change; MAFS, mitral annular fractional shortening; MAP-D, end-diastolic mitral annular perimeter; MAP-S, end-systolic mitral annular perimeter; TOF, tetralogy of Fallot; etrTOF, TOF early total reconstruction; pcTOF, TOF early palliation, late correction.

properties could not be confirmed in pcTOF patients.

\section{Discussion}

According to recent findings from imaging studies, significant alterations could be detected in the left heart including LV and LA morphology and function in addition to right heart abnormalities in adults with repaired TOF (3,4,11-13). Most repaired TOF patients show a normal $\mathrm{LV}$ twisting pattern, but with a significantly lower LV twist mainly due to decreased apical rotation (11). Almost one-third of the subjects had an abnormal twist pattern due to both abnormal apical and/or basal LV rotations. Over a quarter of the patients had abnormal apical rotation that was associated with larger LV dimensions and decreased biventricular systolic function. Regarding to $\mathrm{LV}$ global longitudinal function in repaired TOF, LV global longitudinal strain (LV-GLS) was found to be reduced mainly due to the interventricular septum probably by mechanical coupling of the ventricles (12). In recent 3DSTE-derived atrial studies, both RA and LA volumes seemed to be increased in adult patients with repaired TOF with almost similar deformational abnormalities of RA and LA $(3,4)$.

Mitral valve and its annulus play an important role in regulating blood flow between LV and LA. According to the above-mentioned alterations it could be theorized, that MA could show significant remodelling in repaired TOF. 3DSTE was found to be a valuable tool not only for volumetric and functional assessment of different cardiac chambers $(3,4,10)$, but also for the evaluation of MA dimensions and functional properties respecting the cardiac cycle (8). Although 3DSTE-derived methodology does not assess the 3D spatial saddle shape of MA directly, but only its projection to the chosen 2D plane, dilation of MA dimensions and MA functional impairment could be demonstrated in adult repaired TOF patients, regardless of the type of correction. Moreover, patients who were treated 
with the two-step way (early palliation, late correction) showed worse results suggesting clinical benefits of early total reconstruction on late MA morphology and function. Moreover, correlation between the age of early total reconstruction and MA systolic dimensions could confirm these findings. However, further studies are warranted to confirm our findings in a larger patient population evaluating their prognostic significance, as well.

Several important limitations have arisen during the assessments:

(I) A relatively small number of patients from a single center by a single observer (DP) were examined. However, it should be considered that repaired TOF is a relatively rare disease;

(II) Moreover, adult patients with some risk factors were assessed, therefore their effects could also be taken into considerations when interpreting results;

(III) The current image quality of 3DSTE is inferior to that of $2 \mathrm{D}$ echocardiography due to poor temporal and spatial image resolutions;

(IV) During the present study, ventricular and atrial volumetric and functional characterization was not aimed to be performed.

\section{Conclusions}

MA enlargement and functional impairment could be detected in adult patients with repaired TOF regardless of the type of correction. However, pcTOF patients have worse results.

\section{Acknowledgments}

None.

\section{Footnote}

Conflicts of Interest: The authors have no conflicts of interest to declare.

Ethical Statement: The authors are accountable for all aspects of the work in ensuring that questions related to the accuracy or integrity of any part of the work are appropriately investigated and resolved. All repaired TOF patients and control subjects gave informed consent. The institutional human research committee approved the study which complied with the 1975 Declaration of Helsinki.

\section{References}

1. Swamy P, Bharadwaj A, Varadarajan P, et al. Echocardiographic evaluation of tetralogy of Fallot. Echocardiography 2015;32 Suppl 2:S148-56.

2. Bedair R, Iriart $X$. Educational series in congenital heart disease: Tetralogy of Fallot: diagnosis to long-term followup. Echo Res Pract 2019;6:R9-23.

3. Nemes A, Havasi K, Domsik P, et al. Evaluation of right atrial dysfunction in patients with corrected tetralogy of Fallot using 3D speckle-tracking echocardiography. Insights from the CSONGRAD Registry and MAGYARPath Study. Herz 2015;40:980-8.

4. Havasi K, Domsik P, Kalapos A, et al. Left Atrial Deformation Analysis in Patients with Corrected Tetralogy of Fallot by 3D Speckle-Tracking Echocardiography (from the MAGYAR-Path Study). Arq Bras Cardiol 2017;108:129-34.

5. Nesser HJ, Mor-Avi V, Gorissen W, et al. Quantification of left ventricular volumes using three-dimensional echocardiographic speckle tracking: Comparison with MRI. Eur Heart J 2009;30:1565-73.

6. Saito K, Okura H, Watanabe N, et al. Comprehensive evaluation of left ventricular strain using speckle tracking echocardiography in normal adults: Comparison of threedimensional and two-dimensional approaches. J Am Soc Echocardiogr 2009;22:1025-30.

7. Yu HK, Li SJ, Ip JJ, et al. Right ventricular mechanics in adults after surgical repair of tetralogy of Fallot: insights from three-dimensional speckle-tracking echocardiography. J Am Soc Echocardiogr 2014;27:423-9.

8. Nemes A, Földeák D, Kormányos Á, et al. Cardiac Amyloidosis Associated with Enlargement and Functional Impairment of the Mitral Annulus: Insights from the Three-Dimensional Speckle Tracking Echocardiographic MAGYAR-Path Study. J Heart Valve Dis 2017;26:304-8.

9. Havasi K, Kalapos A, Berek K, et al. More than 50 years' experience in the treatment of patients with congenital heart disease at a Hungarian university hospital. The basics of the CSONGRAD Registry. Orv Hetil 2015;156:794-800.

10. Nemes A, Kalapos A, Domsik P, et al. Three-dimensional speckle-tracking echocardiography - a further step in noninvasive three-dimensional cardiac imaging. Orv Hetil 2012;153:1570-7.

11. Menting ME, Eindhoven JA, van den Bosch AE, et al. 
Abnormal left ventricular rotation and twist in adult patients with corrected tetralogy of Fallot. Eur Heart J Cardiovasc Imaging 2014;15:566-74.

12. Menting ME, van den Bosch AE, McGhie JS, et al. Assessment of ventricular function in adults with repaired Tetralogy of Fallot using myocardial deformation imaging.

Cite this article as: Nemes A, Kormányos Á, Havasi K, Kovács Z, Domsik P, Kalapos A, Hartyánszky I, Ambrus N, Forster T. Mitral annulus is enlarged and functionally impaired in adult patients with repaired tetralogy of Fallot as assessed by three-dimensional speckle-tracking echocardiographyresults from the CSONGRAD Registry and MAGYAR-Path Study. Cardiovasc Diagn Ther 2019;9(Suppl 2):S221-S227. doi: 10.21037/cdt.2019.06.08
Eur Heart J Cardiovasc Imaging 2015;16:1347-57.

13. Timóteo AT, Branco LM, Rosa SA, et al. Usefulness of right ventricular and right atrial two-dimensional speckle tracking strain to predict late arrhythmic events in adult patients with repaired Tetralogy of Fallot. Rev Port Cardiol 2017;36:21-9. 